\title{
Interface Sharpening instead of Broadening by Diffusion in Ideal Binary Alloys
}

\author{
Z. Erdélyi, ${ }^{*}$ I. A. Szabó, and D. L. Beke \\ Department of Solid State Physics, University of Debrecen, P.O. Box 2, H-4010 Debrecen, Hungary
}

(Received 10 June 2002; published 1 October 2002)

\begin{abstract}
We demonstrate, using computer simulations based on deterministic kinetic equations and Monte Carlo technique, that during intermixing in an ideal $A B$ system with an initially wide $A / B$ interfaceif the diffusion coefficient $D$ strongly depends on concentration - the interface can become sharp on nanoscale. The sharp interface shifts proportionally with time (in contrast to the square root law). Furthermore, it is also shown that at the beginning of the intermixing in a finite bilayer or in multilayers, the diminution of the concentration gradient takes place by filling up one of the initially pure layers (layer $B$ if $D$ is large there) and by the shift of the sharpening interface.
\end{abstract}

DOI: $10.1103 /$ PhysRevLett.89.165901

PACS numbers: 66.30.Pa, 68.35.Fx

Very recently, studying the dissolution of thin (3 and 8 monolayers thick) Ni films into $\mathrm{Cu}(111)$ substrate, it was shown (both by experiments and by computer simulations, based on deterministic kinetic equations) that the interface remains sharp and shifts proportionally with the time $t$ (in contrast to a shift with $\sqrt{t}$ ) even in ideal systems having complete mutual solubility [1]. This result is inherently related to the strong nonlinearity of the problem: the strong concentration dependence of the diffusion coefficient $D$ shifts the validity limit of the continuum approach (see also [2]), from which a parabolic law would be expected, out of the nanometer range. It was also shown in [1] that the parabolic law is obeyed at longer times and thus this "irregular" behavior can be observed only on nanoscale. Furthermore, it was obtained from computer simulations in [2] that, at the beginning of the intermixing in a finite bilayer or in multilayers with initially sharp $A / B$ interfaces, the diminution of the concentration gradient takes place by filling up one of the initially pure layers (layer $B$ if $D$ is large there) and by the shift of the sharp interface. Although the above simulations were carried out for a discrete lattice, this phenomenon was also observed experimentally in the amorphous Si-Ge multilayers [3] by Auger depth profiling technique [4], illustrating that the above effects are rather related to the nonlinearity than to the discrete character of the medium, where the diffusion takes place.

In this Letter, using computer simulations based on deterministic kinetic equations (discrete, atomic approach) [5] and Monte Carlo technique (which contains the effects of fluctuations as well), we demonstrate that on nanoscale (short diffusion distances, short time) and again in the case of strongly concentration dependent $D$, an initially wide $A / B$ interface can become sharp even in an ideal system. While such a process is obvious in an alloy with a large miscibility gap (the metastable solid solution in the smeared interface region decomposes and a sharp interface should be formed), it is surprising at first sight in systems with complete mutual solubility, because according to the macroscopic Fick I law the direction of the atomic flux is always opposite to the direction of the concentration gradient. Indeed, for concentration independent $D$, the concentration profile will gradually decay and only a flattening of the (sharp or wide) interface, produced experimentally, is generally expected.

The problem is interesting not only from the point of view of fundamental research. For example, the Ni/Cu system is a model material for giant magnetoresistance, and in these systems the abruptness of the interface, and the knowledge of the possibilities for its improvement, is a key point (e.g., [6]). Furthermore, multilayers made from Mo and V (which is also an ideal binary system) are model materials for $\mathrm{x}$-ray mirrors, or $\mathrm{Si} / \mathrm{Ge}$ systems are basic semiconductor structures, where again the sharpness of the interfaces can be a very important requirement for many applications. It is also well known that in molecular beam epitaxy grown Si-Ge multilayers the $\mathrm{Ge} / \mathrm{Si}$ interface, produced by the deposition of $\mathrm{Si}$ on $\mathrm{Ge}$, is always less sharp (due to the mixing driven by the segregation of $\mathrm{Ge}$ during the growth) than the $\mathrm{Si} / \mathrm{Ge}$ interface $[7,8]$. The effect described here offers a way for the improvement of the multilayers by sharpening of the interface and the diminution of the asymmetry by annealing at moderate temperatures for relatively short times.

In our computer simulations, the deterministic kinetic equations (for the exchange mechanism) were solved in a very similar way as in [1] for the same input parameters: only the initial conditions were different here. The basic equations are the following [5]:

$$
\begin{aligned}
\frac{d C_{i}}{d t}=-z_{v}[ & C_{i}\left(1-C_{i-1}\right) \Gamma_{i, i-1}-\left(1-C_{i}\right) C_{i-1} \Gamma_{i-1, i} \\
& \left.+C_{i}\left(1-C_{i+1}\right) \Gamma_{i, i+1}-\left(1-C_{i}\right) C_{i+1} \Gamma_{i+1, i}\right] .
\end{aligned}
$$

$C_{i}$ is the probability that a site in plane $i$ is occupied by an $A$ atom and $\left(1-C_{i+1}\right)$ gives the probability that one site 
in plane $(i+1)$ is occupied by a $B$ atom $\left(z_{v}\right.$ is the vertical coordination number). The $\Gamma_{i, i+1}$ jump frequencies have an Arrhenius-type temperature dependence:

$$
\Gamma_{i, i+1}=\nu \exp \left(-\frac{E_{i, i+1}}{k_{B} T}\right)
$$

where $\nu$ denotes the attempt frequency. $E_{i, i+1}$ is the activation barrier and in our calculations, following [2,9], it was chosen as:

$$
\begin{aligned}
E_{i, i+1}= & E^{0}-\left[z_{v}\left(C_{i}+C_{i+2}\right)+z_{l} C_{i+1}\right]\left(V_{A B}-V_{B B}\right) \\
& +\left[z_{v}\left(C_{i-1}+C_{i+1}\right)+z_{l} C_{i}\right]\left(V_{A B}-V_{A A}\right) \\
& -Z\left(V_{A B}+V_{B B}\right), \\
i= & 2, \ldots, m-1,
\end{aligned}
$$

which in ideal $\left(2 V_{A B}=V_{A A}+V_{B B}\right.$, where $V_{A A}, V_{B B}$, and $V_{A B}$ are the pair interaction energies between $A-A, B-B$, and $A-B$ atoms), homogeneous systems can be rewritten as [2]

$$
E_{i, i+1}=Q_{0}-m c .
$$

Here $Q_{0}$ is the activation energy in the pure $B$ matrix and $m=Z\left(V_{A A}-V_{B B} / k T\right)\left(Z=2 z_{v}+z_{l}\right)$, where $z_{l}$ is the lateral coordination number). Accordingly, the diffusion coefficients $D$ of the two constituents (measured in such a homogeneous alloy) are equal to each other and have an exponential composition dependence, $D(C)=$ $D(0) \exp (m C)$. For the sake of graphic description, it is worth introducing the $m^{\prime}=m \log e$ parameter, which gives the difference of the orders of magnitudes of the diffusion coefficients in pure $A$ and $B$ matrices. It can be estimated from the Arrhenius functions published in pure $A$ and $B$ matrices and, for example, in $\mathrm{Cu}-\mathrm{Ni}$ and $\mathrm{Si}-\mathrm{Ge}$ systems the experimental value of $m^{\prime}$ is about 4 at $700 \mathrm{~K}$, whereas it is 7.3 for Mo-V system at $1053 \mathrm{~K}[10,11]$. Note that $m^{\prime}$ can also be determined from the known pair interaction energies [2] (from the expression given above for $m$ ).

A numerical method (for the details, see [1]) was used to obtain the time dependence of the concentration in each layer for $T=1000 \mathrm{~K}$, with $V_{A A}=-0.74 \mathrm{eV}$ and $V_{B B}=-0.58 \mathrm{eV}$, i.e., for $m^{\prime} \cong 9$. The calculations were carried out for two different initial conditions: (A) ten atomic layers of $A /$ semi-infinite $B(111)$ substrate and (B) symmetric $A / B$ multilayer with a modulation length of 41 atomic layers. In both cases the interface was eight planes wide with linearly varying composition. Figure 1 shows the dissolution of ten atomic layers of $A$ into a bulk $B(111)$ substrate. It can be seen that at the beginning the initially wide interface becomes more and more sharp. After the sharpening process - as is expected - the dissolution is the same as obtained in [1]: the interface remains atomic sharp and shifts step-by-step (layer-bylayer dissolution mode). The process, shown in Fig. 1, clearly reflects the asymmetry of the diffusion: there is

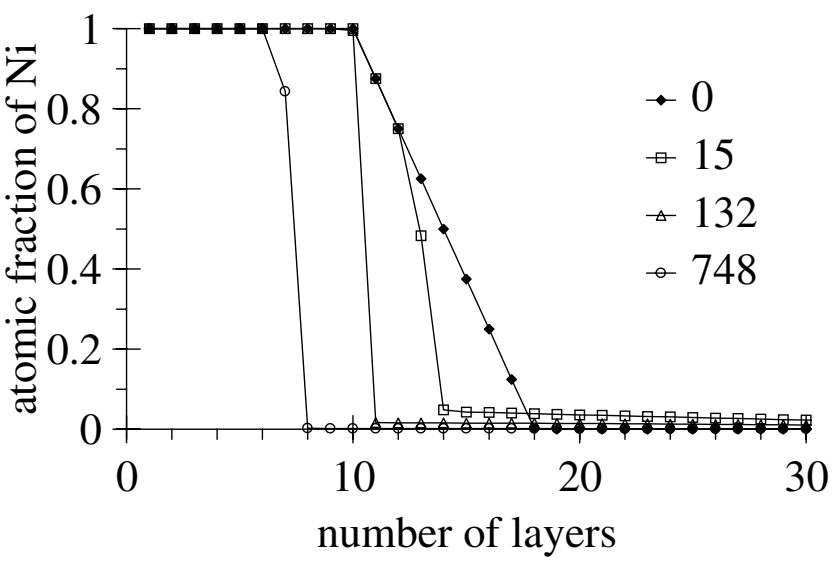

FIG. 1. Composition distribution of $A$ during the dissolution of ten atomic layers of $A$ into $B(111)$ calculated using deterministic kinetic equations. The initially eight atomic layers width interface becomes abrupt and then remains sharp during the whole process due to the asymmetric diffusion (concentration dependent diffusivity). (Time units shown in the figure must be multiplied by 1000.)

practically no diffusion in pure $A$ and there is a very fast diffusion in pure $B$, leading to a gradual sharpening of the composition profile.

As was already mentioned, an originally abrupt interface can remain sharp during diffusional intermixing in multilayers as well. Therefore, it is an interesting question whether an originally wide interface can also become sharper in multilayers. The situation differs from the dissolution of a thin film into a semi-infinite substrate because in this case the finite character of the system can influence the dissolution process. The most important effect is that the $B$ layer can be saturated by $A$ atoms and this leads to the change of the diffusivity there. However, as Fig. 2 shows, the first part of the process is the interface sharpening in this case as well. At longer times the interface shifts step-by-step and just after the consuming of $A$, the complete homogenization takes

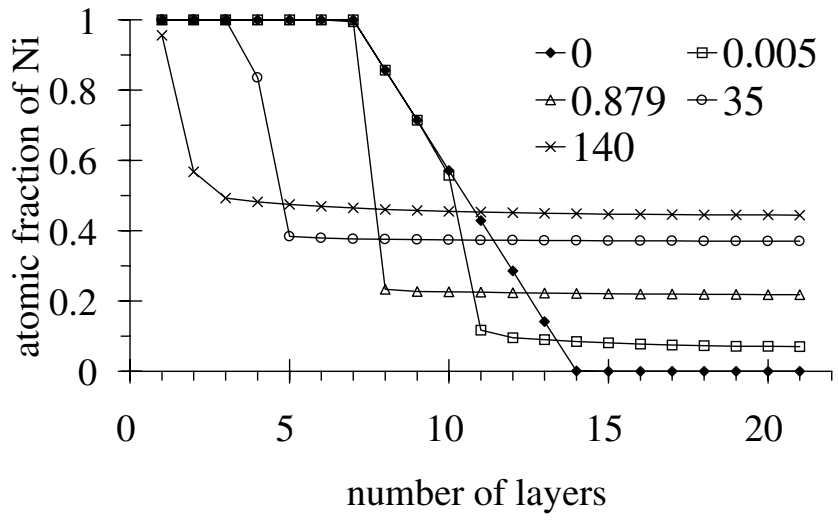

FIG. 2. Interface sharpening in an $A / B$ multilayer system. Only a half-bilayer is shown in the figure because of an $A$ or $B$ monolayer is symmetric to its center. (Time units shown in the figure must be multiplied by $10^{6}$.) 
place. This result also gives a plausible explanation for the apparent contradiction with the continuum Fick I law:

$$
j=-D \operatorname{grad} C
$$

Since in ideal systems $D$ has a positive value, the direction of the flux is always opposite to the direction of the concentration gradient and, for concentration independent diffusion coefficients, this equation should lead to flattening of the interface. However, if $D=D(c), j$ depends not only on the concentration gradient but also on the local composition of the sample. Figure 3 illustrates the "flux distribution" at the interface in the initial state, when the film and the substrate are separated by a wide interface. As the concentration gradient is constant along the interface, regarding Eq. (5), it is only $D$ on which the absolute value of the atomic flux depends. Therefore, in the case of concentration independent $D$, the atomic fluxes, independently of the position, are the same, whereas in the case of $D=D(c)$, the flux distribution follows the $D=D(c)$ function (Fig. 3). Thus, in principle, even the continuum flux equation is capable to describe some sharpening.

Obviously at longer annealing times - as is expected from general thermodynamics - the homogenization should take place. It can be clearly seen that indeed this is the case for the multilayer sample: although at the beginning the process decreases the gradient by filling up of $B$ with $A$ (and not by flattening of the interface), the final state is the completely intermixed homogeneous alloy. For the case of semi-infinite geometry, the first part of the intermixing (the initial sharpening and linear shift of the interface) will extend to times under which the deposited film is consumed. Of course, for thick films, before reaching this stage, the kinetics of the dissolution will gradually change from linear to parabolic [1], and this transition time will be determined by the "strength"

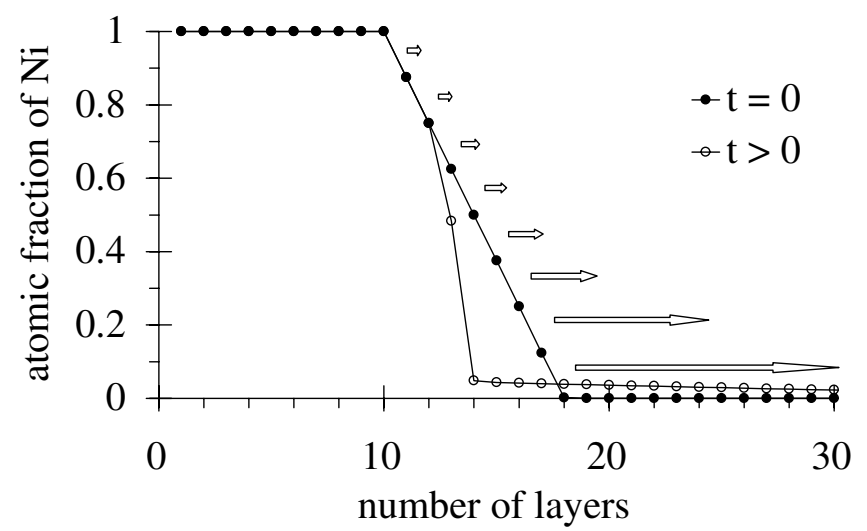

FIG. 3. Schematic drawing of the flux distribution in the initial state $(t=0$ - solid circles) in the case of concentration dependent diffusion coefficients. The arrows represent the atomic flux and its lengths are proportional to the absolute value of the flux. of the concentration dependence of the diffusion coefficients, $m^{\prime}$. For $m^{\prime} \cong 0$ the "normal" intermixing with the formation of a symmetrical diffusion profile will take place, while with increasing value of $m^{\prime}$, the diffusion profile will be more and more asymmetrical and, finally, the above discussed effects can be observed on nanoscale. It is important to note that $m^{\prime}$ is inversely proportional to the temperature, and thus with decreasing temperature it is easy to reach those values for which the above nonlinear effects can be observed. Indeed-as can be checked from the known pair interaction energies or from the experimental values of $D$-it is very general that at low temperatures $m^{\prime}$ is large enough that the sharpening of the interface is quite generally expected.

In order to take into account the role of fluctuations (stochastic processes), we have repeated the above computer calculations by Monte Carlo simulations as well, with the same assumptions as in the layered deterministic model; i.e., it was supposed that: (a) the diffusion is modeled with a direct exchange mechanism; (b) the exchange frequency depends only on the total number of $A$ neighbors for an $A B$ pair according to the nearest neighbor interaction approximation; (c) the saddle point energy is constant. This jump frequency model represents a random alloy, since the exchange frequency depends only on the total number of $A$ and $B$ neighbors; i.e., the forward and backward jumps have the same probability. Therefore, there is no tendency for ordering or clustering. Furthermore, we have a concentration dependent diffusivity, since the jump frequency is increasing with the number of $A$ neighbors. The same input parameters were used as in the case of the deterministic calculations. Figure 4 shows that this computer simulation resulted in the same behavior; i.e., the interface became sharp in this case as well.

It is worth noting that, although our calculations are based on direct exchange of atoms, the above conclusions

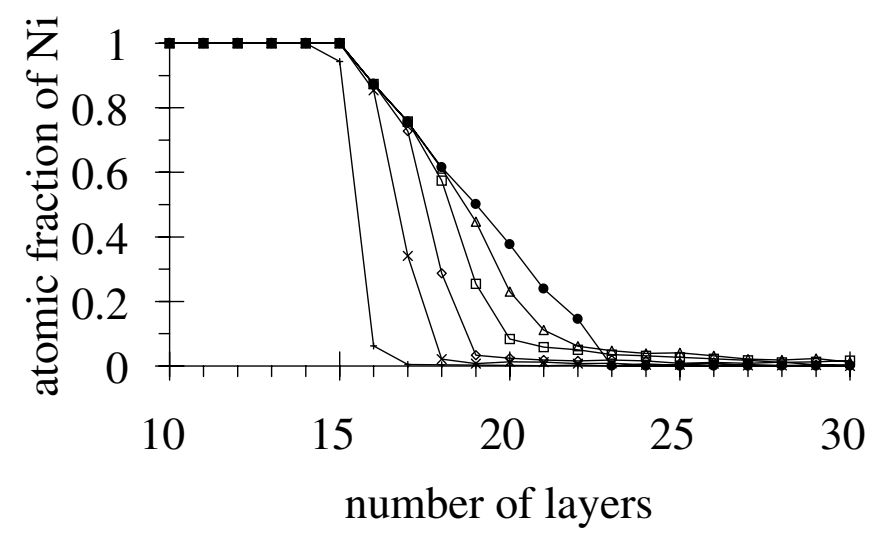

FIG. 4. Dissolution of 15 atomic layers of $A$ into $B(111)$ calculated using the Monte Carlo method. Qualitatively the same phenomenon can be observed as in Fig. 1. (Compositions are calculated by averaging the composition of each atomic layer perpendicular to the direction of diffusion.) 
are independent of the mechanism of diffusion (see also the comments to Fig. 3); i.e., they are valid, e.g., for vacancy mechanism as well.

In summary, we have shown that, in the case of a concentration dependent diffusion coefficient, the diffusion mixing can lead to sharpening of the interface in systems having a complete mutual solubility as well. The atomic flux depends not only on the concentration gradient but also on the concentration dependent diffusion coefficient. Consequently, the atomic flux is not constant for a constant concentration gradient, either. This can lead to interface sharpening, which also takes place if the stochastic character of the process (in the Monte Carlo simulation) is considered.

This work was supported by the OTKA Board of Hungary (No. T038125). The authors are indebted to Hans Bakker for enlightening discussions.

*Electronic address: zerdelyi@dragon.klte.hu
[1] Z. Erdélyi, C. Girardeaux, Z. Tókei, D. L. Beke, C. Cserháti, and A. Rolland, Surf. Sci. 496, 129 (2002).

[2] Z. Erdélyi, D. L. Beke, P. Nemes, and G. A. Langer, Philos. Mag. A 79, 1757 (1999).

[3] A. Csik, G. A. Langer, D. L. Beke, Z. Erdélyi, M. Menyhárd, and A. Sulyok, J. Appl. Phys. 89, 804 (2001).

[4] A. Barna and M. Menyhárd, Phys. Status Solidi A 145, 263 (1994).

[5] G. Martin, Phys. Rev. B 41, 2279 (1990).

[6] X.W. Zhou et al., Acta Mater. 49, 4005 (2001).

[7] D. E. Jesson, in Handbook of Thin Film Process Technology, edited by D. A. Glocker and S. I. Shah (IOP Publishing Ltd., Bristol and Philadelphia, 1997), Vol. F, p. F1:1.

[8] T. Walther, C. J. Humphreys, and D. J. Robbins, Defect Diffus. Forum 143-147, 1135 (1997).

[9] C. Cserháti, H. Bakker, and D. L. Beke, Surf. Sci. 290, 345 (1993).

[10] W. F. Egelhoff, J. Vac. Sci. Technol. A 7, 2060 (1989).

[11] H. Mehrer, Diffusion in Solid Metals and Alloys, Landolt-Bornstein New Series Vol. III/26 (Springer, Berlin, 1990). 